\title{
Editorial
}

Nephrology

\section{Periostin: The Missing Link to Attenuate Kidney Fibrosis}

\author{
Niki Prakoura ${ }^{a}$ Christos Chatziantoniou ${ }^{a, b}$ \\ ${ }^{a}$ Institut National de la Santé Et de la Recherche Médicale UMRS 1155, Tenon Hospital, Paris, France; \\ bSorbonne Université, UPMC Paris 6, Paris, France
}

Chronic kidney disease (CKD) is a major risk factor for end-stage renal disease and death, affecting almost $10 \%$ of the world population. With a continuously growing incidence and no available prognostic or therapeutic treatments, CKD currently represents an important societal and economic burden for modern healthcare systems [1]. Renal replacement therapy through dialysis or transplantation remains the only available option for treatment of end-stage renal disease.

CKD can originate from different pathophysiologic conditions, including diabetes, hypertension and immune or toxic aggressions of the kidney. Independent of the initial cause, there are common pathways involved in the progression of CKD, such as chronic inflammatory responses and development of fibrosis which drive the impairment of renal function. Fibrosis occurs because of a chronic or deregulated wound repair process, frequently in response to a continuous or repetitive insult. During the last years, many efforts were made to unravel novel mediators and targets for therapy of renal disease. Despite the identification of several factors mediating renal inflammation and fibrosis, including different growth factors (transforming growth factor- $\beta$ [TGF- $\beta$ ], connective tissue growth factor, platelet-derived growth factors [PDGFs], angiotensin-II) or cytokines (monocyte chemoattractant protein-1 [MCP-1], tumor necrosis factor alpha), however, no specific treatment has yet been established to prevent fibrosis [2].

\section{KARGER}

(๑) 2017 S. Karger AG, Basel

E-Mail karger@karger.com

www.karger.com/ajn
Periostin is a recently identified novel biomarker and/ or mediator of CKD. Periostin is a $90 \mathrm{kDa}$ secreted matricellular protein abundant in bone and dental tissues. Interestingly, although its expression is scarce in mature organs, it is highly induced after tissue injury. Periostin was shown to interact with other extracellular matrix components, such as collagen I, tenascin-C, fibronectin and BMP-1, promoting collagen fibrilogenesis and altering the biomechanical properties of connective tissues. Apart from its association with matrix proteins, periostin is believed to trigger intracellular signaling through interaction with cell-surface integrins, thus modulating cell adhesion, migration, proliferation and differentiation.

Periostin promotes inflammation and fibrosis in different disease states involving several organs. These include myocardial infarction, cardiac hypertrophy, idiopathic pulmonary diseases, asthma, skin sclerosis, hepatic fibrosis, muscular dystrophy and renal diseases. An increasing number of studies, over the last years, have highlighted the involvement of periostin in the progression of CKD. Periostin is de novo expressed in biopsies of patients with different renal diseases, including focal segmental glomerulosclerosis, IgA nephropathy, diabetes, lupus nephritis and polycystic kidney disease. Interestingly, the localization of periostin in the damaged tissue was associated with sites of periglomerular, vascular or interstitial fibrosis, while its expression levels were correlated with the deterioration of renal structure and function [3].

Dr. Christos Chatziantoniou INSERM UMRS 1155 Tenon Hospital, 4 rue de la Chine FR-75020 Paris (France)

E-Mail christos.chatziantoniou@upmc.fr 
Studies in animal models of renal disease unraveled important aspects regarding the role of periostin in the progression of renal pathology. An initial study involving an L-NAME-induced model of hypertensive nephropathy coupled with administration of an angiotensin-II receptor blocker revealed tissue periostin as a potential target of interest against renal disease development [4]. Subsequently, other studies have shown periostin to mediate both inflammation and fibrosis in animal models of unilateral ureteral obstruction (UUO) and nephrotoxic serum-induced glomerulonephritis. Either use of mice lacking periostin or administration of antisense oligonucleotides against periostin inhibited the progression of the disease and preserved renal structure and function $[5,6]$. In a polycystic kidney disease mouse model, periostin was highly expressed by cyst-lining epithelial cells and shown to promote epithelial cell proliferation, autophagy and interstitial fibrosis [7]. Expression of periostin may be induced in different disease contexts by diverse factors or signaling pathways, such as TGF- $\beta 1$, angiotensin-II, PDGF-B, interleukin (IL) 4 , IL13, Ras/cAMP response element-binding protein, extracellular signal-regulated kinase and nuclear factor-kappa B. Moreover, except for stabilizing extracellular matrix interactions and mediating cross-linking of collagen, periostin interacts with integrins $\alpha v \beta 3$ and $\alpha v \beta 5$ to activate PI3 kinase/Akt and Focal Adhesion Kinase pathways, promoting cell adhesion, motility and proliferation [3].

The study of Hwang et al. [8] published in this issue pursues previous results to show that experimental inhibition of periostin with a specific neutralizing antibody can be efficient in attenuating the progression of renal fibrosis. In the first part of their study, the authors verify that the lack of periostin in knock-out mice is protective against the development of renal inflammation and fibrosis in the UUO model, in their experimental setting. The broad investigation of different profibrotic mediators demonstrated that the induction of several key molecules, including collagen I, fibronectin, TGF- $\beta, \alpha$-SMA and Snail was significantly ameliorated in periostin knockout mice. In parallel, the expression of periostin and its potential integrin receptors, $\alpha v \beta 3$ and $\alpha v \beta 5$, was highly induced in wild-type UUO mice. Interestingly, the expression of integrin $\alpha v$ and integrin $\beta 3$ was also attenuated in knock-out mice. Moreover, the infiltration of macrophages, lymphocytes and neutrophils together with the expression of proinflammatory mediators was largely reduced in mice lacking periostin.

Next, the authors proceeded to investigate the effect of periostin treatment alone or in combination with TGF- $\beta$ in an in vitro culture system of primary renal tubular cells.
The results demonstrated that either recombinant periostin or recombinant TGF- $\beta$ alone or in synergy could induce the expression of both periostin and $\alpha$-SMA, which implies a connection between the expression of periostin and induction of a mesenchymal phenotype in renal epithelial cells. Moreover, treatment with either periostin or TGF- $\beta$ induced a differential expression of the integrin subunits $\alpha v, \beta 3$ and $\beta 5$. Interestingly, treatment of the cells with an integrin inhibitor attenuated the induction of colla1, fibronectin and $\alpha$-SMA in response to the synergistic treatment of TGF- $\beta$ and periostin, which indicates the involvement of integrin signaling in the fibrosisrelated pathways induced by periostin and TGF- $\beta$.

Finally, the investigators use a commercial anti-periostin antibody to block the function of periostin in the UUO model for the first time. Administration of the antiperiostin antibody significantly attenuated the progression of fibrosis, as demonstrated by the decreased accumulation of collagen and the reduction in the expression of fibronectin and a-SMA. Importantly, periostin blockade not only inhibited development of fibrosis but showed a broad influence in the progression of the disease. This was a consequence of macrophage infiltration and apoptosis of renal tubular cells being reduced in anti-periostin treated mice, coupled with decreased expression of proinflammatory MCP-1 and proapoptotic p53. Moreover, in vitro studies in cultured primary tubular cells showed that treatment with the anti-periostin antibody could directly inhibit the upregulation of colla1, fibronectin and a-SMA mRNAs induced by recombinant periostin.

Although the involvement of periostin in CKD progression was demonstrated in diverse animal models of renal disease, targeting periostin in human CKD has not been pursued mainly because there are no validated drugs using it as a target in man. The study by Hwang et al. [8] nicely demonstrates that periostin blockade using a neutralizing antibody is a promising therapeutic option to prevent or slow CKD progression and paves the way for pursuing these results in future preclinical or clinical studies.

\section{Acknowledgments}

This work was financially supported by funds from Institut National de la Santé Et de la Recherche Médicale (INSERM) and the Agence Nationale de la Recherche.

\section{Disclosure Statement}

The authors have declared that no conflict of interest exists. 


\section{References}

1 Naghavi M, Wang H, Lozano R, Davis A, Liang $\mathrm{X}$, Zhou $\mathrm{M}$, et al: Global, regional, and national age-sex specific all-cause and causespecific mortality for 240 causes of death, 1990-2013: a systematic analysis for the Global Burden of Disease Study 2013. Lancet 2015; 385:117-171.

2 Lee SY, Kim SI, Choi ME: Therapeutic targets for treating fibrotic kidney diseases. Transl Res 2015; 165:512-530.

-3 Prakoura N, Chatziantoniou C: Periostin in kidney diseases. Cell Mol Life Sci 2017;74: 4315-4320.
Guerrot D, Dussaule JC, Mael-Ainin M, XuDubois YC, Rondeau E, Chatziantoniou C, Placier S: Identification of periostin as a critical marker of progression/reversal of hypertensive nephropathy. PLoS One 2012;7:e31974.

Mael-Ainin M, Abed A, Conway SJ, Dussaule JC, Chatziantoniou C: Inhibition of periostin expression protects against the development of renal inflammation and fibrosis. J Am Soc Nephrol 2014;25:1724-1736.

Prakoura N, Kavvadas P, Kormann R, Dussaule JC, Chadjichristos C, Chatziantoniou C: NFkB-Induced periostin activates integrin- $\beta 3$ signaling to promote renal injury in GN. J Am Soc Nephrol 2017;28:1475-1490.

Wallace DP, White C, Savinkova L, Nivens E, Reif GA, Pinto CS, Raman A, Parnell SC, Conway SJ, Fields TA: Periostin promotes renal cyst growth and interstitial fibrosis in polycystic kidney disease. Kidney Int 2014;85: 845-854.

-8 Hwang JH, Yang SH, Kim YC, Kim JH, An JN, Moon KC, Oh YK, Park JY, Kim DK, Kim YS, Lim CS, Lee JP: Experimental Inhibition of Periostin Attenuates Kidney Fibrosis. Am J Nephrol 2017;46:501-517. 